\title{
Manifestations of Tuberculosis in Ear, Nose, Throat, Head and Neck Region - A Retrospective Study
}

https://doi.org/10.47210/bjohns.2020.v28i2.332

\author{
Vandana P Thorawade, ${ }^{1}$ S. A. Jaiswal, ${ }^{1}$ Seema Ramlakhan Gupta ${ }^{1}$
}

\begin{abstract}
$\underline{\text { Introduction }}$
Tuberculosis can involve any organ or site. Otorhinolaryngologist may encounter tuberculosis affecting lymph nodes, ear, larynx, deep neck spaces, salivary glands etc. which can mimic other chronic granulomatous conditions or malignancy. To ensure early diagnosis, it is important to recognize its cardinal signs and symptoms and to be aware of potential pitfalls in diagnosis. This study was done to learn the clinical presentation of tuberculosis in ear, nose, throat and head and neck region, and to assess the effectiveness of various investigations and treatment done for the same.

Materials and Methods

A retrospective study done in our institution involving 120 patients suffering from tuberculosis in ear, nose, throat and head and neck region who attended pulmonary medicine or ENT OPD or ward between January 2008 to December 2017 that is, 10 years. Study period for data collection and analysis was 1 month.

$\underline{\text { Results }}$

Total 120 patients-69 males and 51 females. Most common site was cervical lymph nodes(77.5\% patients), followed by larynx $(8.3 \%)$,middle ear(7.5\%), deep neck spaces(2.5\%) and salivary glands and nose(1.7\% each). Histopathology was highly sensitive(99. 1\%).All except one patient responded to first-line antitubercular drugs, the other patient was given treatment for $M D R-T B$ to which he responded.

Conclusion

Tuberculosis can involve any site in the head and neck region, most common being cervical lymph nodes mainly presenting as neck swelling. Variable nature of manifestations of tuberculosis makes it essential to have high degree of suspicion for early diagnosis.

Kevwords

Tuberculosis; Otorlaryngology; Lymph Nodes; Larynx; Ear, Middle; Neck; Retrospective Study
\end{abstract}

$\underline{\text { ABSTRACT }}$

$\mathrm{T}$ Tuberculosis (TB) is one of the oldest diseases of mankind and it is very common in developing nations such as India. There are nearly 9 million new patients and 2 million deaths from tuberculosis worldwide every year. ${ }^{1}$ India has about $23 \%$ of world's share. ${ }^{1}$ Tuberculosis is a chronic infectious granulomatous disease caused by Mycobacterium tuberculosis. M. Tuberculosis is an acid fast bacillus. Increased incidence

1 - Department of ENT, Dr. Vaishampayan Memorial Government Medical college, Solapur

\section{Corresponding author:}

Dr Seema Ramlakhan Gupta

email: mailmeseema.gupta5@gmail.com of tuberculosis in India is due to increased prevalence of immunodeficiency through HIV infection, malignancies, drug addiction, poverty and overcrowding.

Health care givers, TB contacts, prisoners, smokers, elderly, slum dwellers form the other socially vulnerable groups. Although TB mainly affects the lungs, extrapulmonary tuberculosis is also a significant disease entity. Among the total 1.68 million patients of TB notified in the year 2014, 2.75 lacs patients were of extrapulmonary tuberculosis. ${ }^{1}$ Tuberculosis can affect any organ in the body except nail, hair and teeth. ${ }^{2}$ In the head and neck region, TB can involve the cervical lymph nodes, larynx, middle ear, nose, oral cavity, salivary 
Table I: Site of tuberculosis

\begin{tabular}{|c|c|c|}
\hline SITE OF TUBERCULOUS LESION & NUMBER OF PATIENTS & PERCENTAGE \\
\hline Cervical lymph nodes & 93 & $\mathbf{7 7 . 5 0} \%$ \\
\hline Larynx & 10 & $\mathbf{8 . 3 0} \%$ \\
\hline Middle ear & 7 & $\mathbf{5 . 8 3} \%$ \\
\hline Nose & 2 & $\mathbf{1 . 6 7 \%}$ \\
\hline Parotid gland & 2 & $\mathbf{1 . 6 7 \%}$ \\
\hline Thyroid gland & 1 & $\mathbf{0 . 8 3} \%$ \\
\hline Deep neck spaces & 3 & $\mathbf{2 . 5 0} \%$ \\
\hline Cervical lymph nodes + middle ear* & 2 & $\mathbf{1 . 6 7 \%}$ \\
\hline
\end{tabular}

*Two patients of middle ear tuberculosis also had cervical lymphadenopathy.

and thyroid glands, deep neck spaces and pharynx. Tuberculosis of lymph node is a common cause of lymph node enlargement. TB can affect middle ear and presents as painless recurrent otorrhoea, presence of abundant pale granulations and severe hearing loss sometimes with facial nerve palsy. ${ }^{3}$ Head and neck tuberculosis is also an interesting field of research because of the varied presentations and different sites of involvement. It may simulate malignancy and may be misdiagnosed. ${ }^{2}$ The various diagnostic methods are direct microscopy, standard culture methods, rapid culture method and polymerase chain reaction test also known as cartridge based nucleic acid amplification test (CB-NAAT). CBNAAT is a rapid diagnostic test which provides results within 2-3 hours and also gives result on rifampicin susceptibility. ${ }^{4}$ Government has proposed an END TB strategy. The important components of this strategy are early diagnosis with latest diagnostic tools, complete treatment with standard antiTB regimen, treatment of comorbidities and providing patient support. ${ }^{5}$ The purpose of this study are to study the clinical presentations of tuberculosis in ear, nose, throat and head and neck regions and to assess the investigations and outcome of anti-Koch's treatment for the same.

\section{Materials and Methods}

It is a retrospective study, the study source being patients attending ENT OPD and ward, pulmonary medicine OPD and ward at our hospital between January 2008 and December 2017, that is, last 10 years. All patients who were diagnosed with tuberculosis of ear, nose, throat and head neck region were included in the study.

Table II: Age distribution

\begin{tabular}{|c|c|c|}
\hline AGE (YEARS) & NUMBER OF PATIENTS & PERCENTAGE \\
\hline $0-20$ & 31 & $25.8 \%$ \\
\hline $20-30$ & 37 & $30.8 \%$ \\
\hline $30-40$ & 19 & $15.8 \%$ \\
\hline $40-50$ & 25 & $20.8 \%$ \\
\hline $50-60$ & 02 & $1.67 \%$ \\
\hline$>60$ & 06 & $5 \%$ \\
\hline
\end{tabular}


Institutional Ethics Committee (IEC) permission was obtained prior to the commencement of the study. We collected patient's data from medical record section and computerized data system. We studied patient's complete history, findings of ear, nose, throat, along with systemic and general examinations. We also reviewed investigations done, e.g., fine needle aspiration cytology and ultrasonography of neck swelling, ZiehlNeelsen staining and culture of ear discharge, if present; biopsy and histopathological examination and also the management of these patients. We then analysed the whole data and compared it with standard observations.

\section{Results}

Total 120 patients of tuberculosis of ear, nose, throat and head-neck region were found and were analysed. (Table I) Most common site was cervical lymph nodes.

Most of the patients of tuberculous lymphadenitis were in age group of 7-25 years with about 64 patients being In this age group; while laryngeal tuberculosis was seen frequently in 40-60 years of age. (Table II)

Out of 120 patients, 69 patients were males and 51 were females.

The chief complaint with cervical lymph node tuberculosis (93 patients) was neck swelling (Fig.1A \&1B) which was unilateral in 86 and bilateral in 7 patients. Level II was most commonly affected (57 patients) followed by level V (26 patients). These patients also had other complaints including fever (22 patients), weight loss (17 patients) and cough with expectoration (8 patients). Fine needle aspiration cytology (FNAC) and ultrasonography USG) were done in all patients. All except four showed tuberculous lymphadenitis on FNAC. In these 4 patients, excisional biopsy of lymph node was done for histopathological diagnosis. Contrast enhanced computed tomography (CECT) neck showed presence of nodal abscess with nodal mass in few patients (Fig. 2). Montoux test was positive in majority of patients (88 patients). Erythrocyte sedimentation rate (ESR) was raised in 75 patients. Eight of these 93 patients had concomitant pulmonary tuberculosis. Six patients were HIV positive.

Patients with laryngeal tuberculosis (10 patients) mainly presented with hoarse voice. All these patients had pulmonary tuberculosis as well. Diagnosis was done by laryngoscopy and biopsy of the lesion for histopathological confirmation. Most common sites in larynx were inter arytenoid area, vocal cords and arytenoids. No patient of laryngeal tuberculosis was found to be HIV positive.

All patients with tuberculosis of middle ear (9 patients) presented with painless profuse otorrhea and 5 of these patients had severe to profound hearing loss at presentation. Two patients had developed ipsilateral mastoid abscess, 1 of which also developed ipsilateral lower motor neuron facial nerve palsy(Fig.1A \&1B). One patient of middle ear tuberculosis had ipsilateral lower motor neuron facial nerve palsy without mastoid abscess. Three patients had multiple perforations (Fig.3), while rest had total perforation. In one patient, the middle ear tuberculosis complicated into tuberculous meningitis. Two patients of middle ear tuberculosis also had cervical lymphadenopathy. Pulmonary tuberculosis was present in all the patients. Ear swab for acid fast bacillus was positive in 7 patients. The 2 patients with mastoid abscess required mastoid exploration and while in 1 patient with facial nerve palsy without mastoid abscess, transmastoid facial nerve decompression was done. Histopathological diagnosis of tuberculosis could be made in all these 3 patients. Sample for histopathology was pale granulations collected while doing mastoid exploration.

Patients with nasal tuberculosis ( 2 patients) presented with blood stained nasal discharge and nasal blockage. They were diagnosed by nasal endoscopy and biopsy of inflammatory nasal tissue. One of these two patients had pulmonary tuberculosis as well.

Patients of parotid gland tuberculosis (2 patients) presented with swelling over parotid region (Fig.4). Among these 2 patients, one developed spontaneous rupture of the parotid resulting in parotid fistula and purulent discharge from that site. Both the patients had tuberculous changes in lungs.

Patient of thyroid gland TB (1 patient) presented with thyroid swelling and the diagnosis was done by FNAC. Patient had tuberculosis of lungs and intestine too. Deep neck space tuberculosis patients (3 patients) 


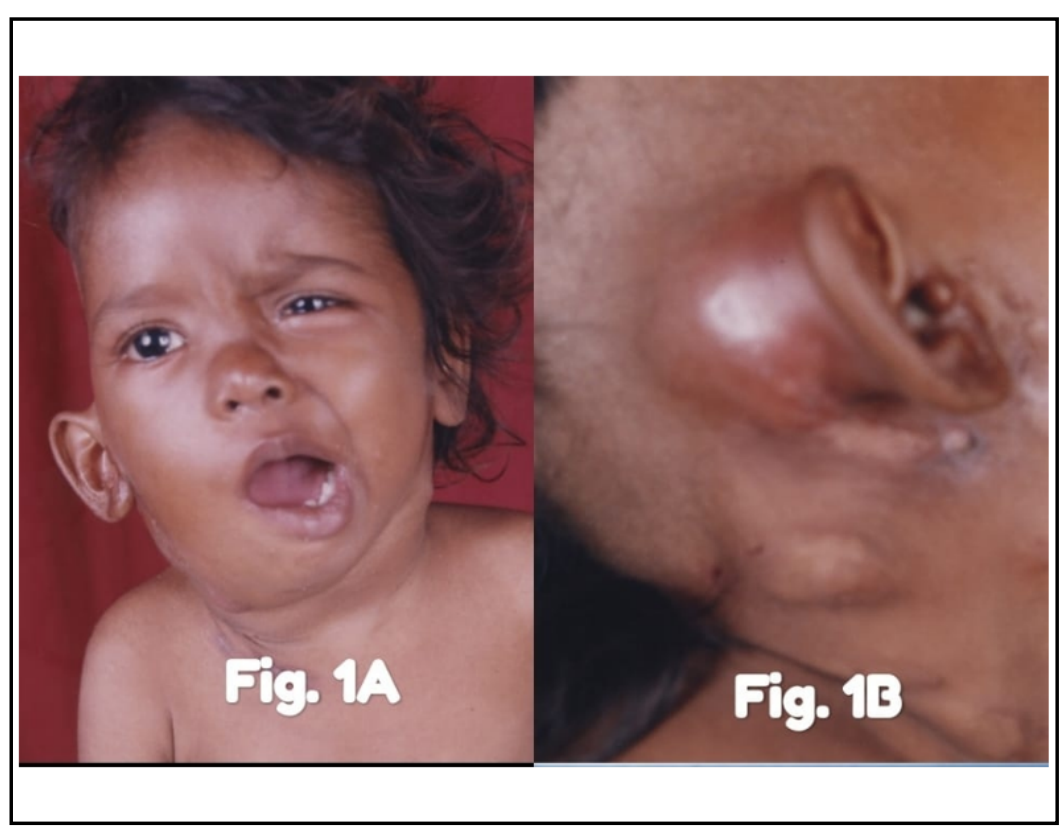

Fig. 1 A \& B. Tubercular mastoid abscess with lower motor neuron in facial palsy with tubercular cervical lymphadenitis

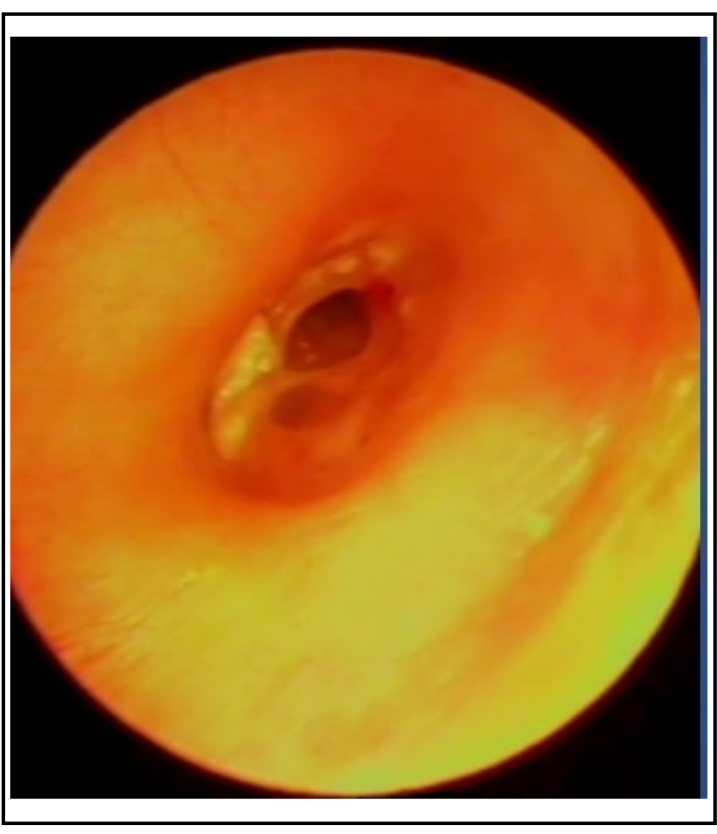

Fig.3. Multiple perforations in tympanic membrane came with complaints of dysphagia and neck pain and TB was diagnosed by microbiological and cytological examinations of the aspirated pus. One of these three

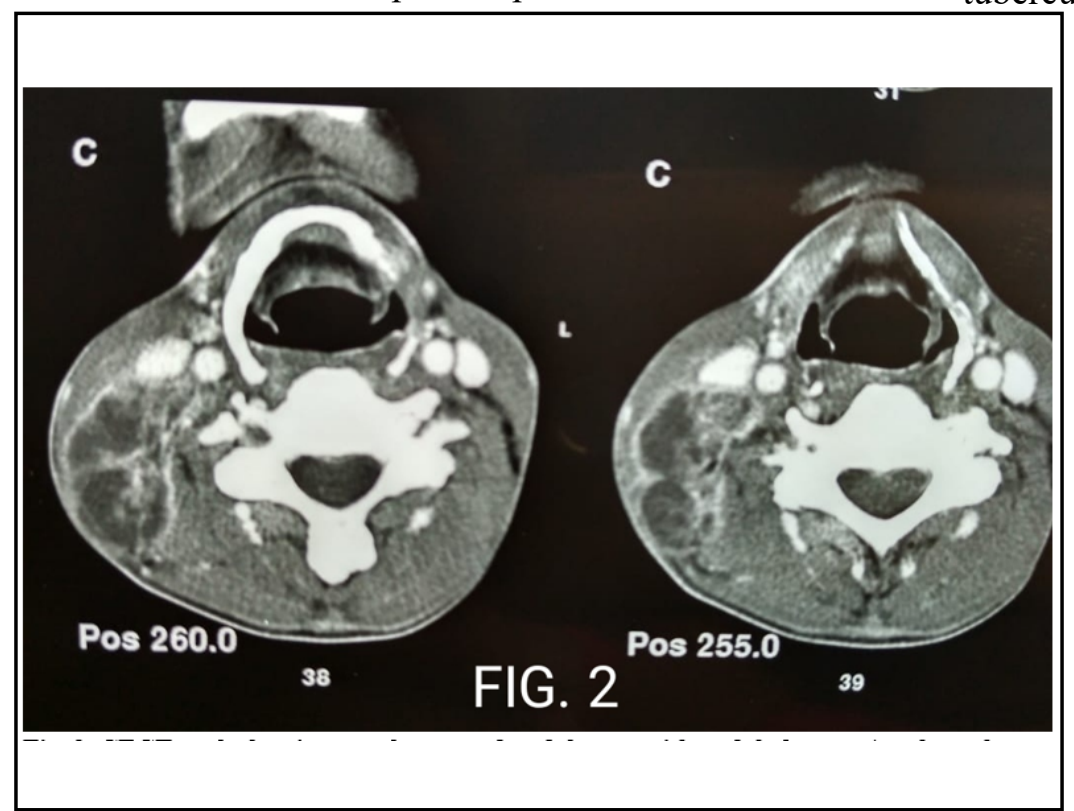

Fig. 2. CECT neck showing conglomerated nodal mass with nodal abscess s/o tuberculous cervical lymphadenopathy patients had pulmonary TB.

Among all these 120 patients of extrapulmonary tuberculosis,32 patients had pulmonary tuberculosis as

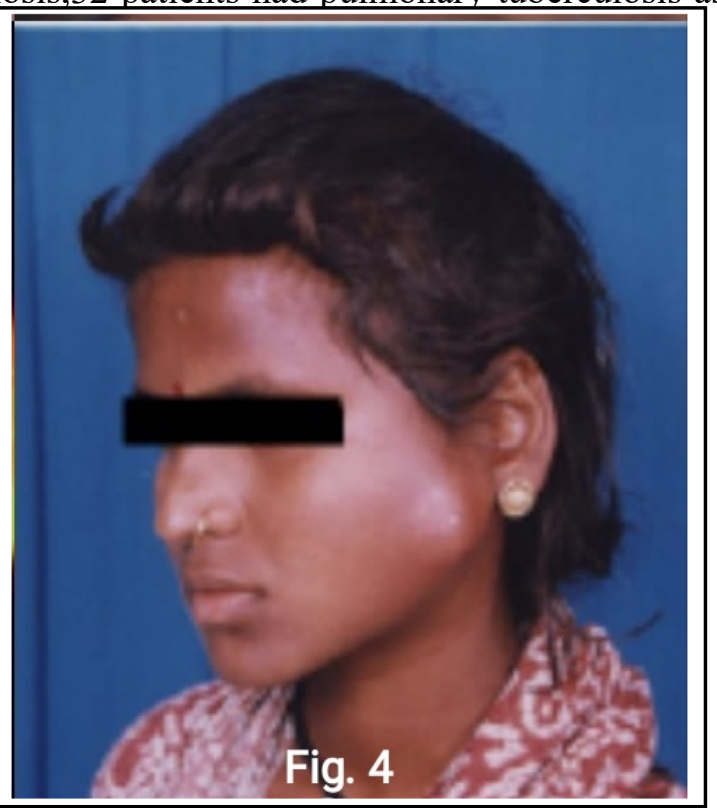

Fig.4. Parotid gland swelling 
Table III: Comparison of the present study with other studies

\begin{tabular}{|c|c|c|c|c|c|c|}
\hline & $\begin{array}{l}\text { PRESENT } \\
\text { STUDY } \\
\text { (2008-17) }\end{array}$ & $\begin{array}{c}\text { PANDURANG } \\
\text { ET AL. (2011- } \\
13)^{18}\end{array}$ & $\begin{array}{c}\text { HAFEEZ } \\
\text { ET AL. } \\
(2005-06)^{19}\end{array}$ & $\begin{array}{l}\text { KHAN ET } \\
\text { AL. (1987- } \\
\qquad 88)^{3}\end{array}$ & $\begin{array}{c}\text { SRIRAM ET } \\
\text { AL. }(2012- \\
14)^{15}\end{array}$ & $\begin{array}{c}\text { DAS ET AL. } \\
\text { (STUDY } \\
\text { PERIOD: } \\
\text { NA) }{ }^{20}\end{array}$ \\
\hline No. of patients & 120 & 70 & 100 & 135 & 104 & 63 \\
\hline Lymph node & $77.50 \%$ & $77 \%$ & $97 \%$ & $79.70 \%$ & $86.50 \%$ & $90.50 \%$ \\
\hline $\begin{array}{c}\text { Deep neck } \\
\text { spaces }\end{array}$ & $2.50 \%$ & $10 \%$ & $2 \%$ & $6.80 \%$ & $3.80 \%$ & \\
\hline Larynx & $8.30 \%$ & $8.50 \%$ & $1 \%$ & $8.30 \%$ & $4.80 \%$ & $7.90 \%$ \\
\hline Salivary glands & $1.70 \%$ & $3 \%$ & & & $3.80 \%$ & \\
\hline Nose & $1.70 \%$ & & & $2.90 \%$ & & \\
\hline Middle ear & $7.50 \%$ & & & $1.96 \%$ & & $1.60 \%$ \\
\hline Pharynx & $\mathbf{0}$ & $1.50 \%$ & & & $1 \%$ & \\
\hline Thyroid gland & $0.83 \%$ & & & & & \\
\hline $\begin{array}{c}\text { Presence of } \\
\text { pulmonary TB }\end{array}$ & $26.67 \%$ & $33 \%$ & & $28.90 \%$ & $16.30 \%$ & \\
\hline $\begin{array}{l}\text { Presence of } \\
\text { HIV }^{\mathrm{a}} \text { infection }\end{array}$ & $5 \%$ & $12.80 \%$ & & & $26 \%$ & \\
\hline
\end{tabular}

${ }^{a} H I V=H u m a n$ Immunodeficiency Virus

well. Chest x-ray changes for pulmonary tuberculosis was seen in all these 32 patients $(26.67 \%)$ while sputum for acid fast bacillus was positive in 27 patients $(22.5 \%)$. All patients were started on anti-Koch's treatment (AKT) according to Revised National Tuberculosis Control Program guidelines and all of them responded well to the first line antituberculous drugs; except 1 patient who had to be given treatment for Multi drug resistant-TB to which he responded. Patients who were positive for HIV were started on anti-retroviral therapy, if not already taking it. No patient died in 1 year follow up period.

\section{Discussion}

Tuberculous cervical lymphadenopathy is the most common presentation of head and neck tuberculosis. ${ }^{6}$ Tuberculous bacilli are carried to the lymph nodes commonly by lymphatics and occasionally by blood route. In lymphatic spread, tubercles are first formed in the cortex while in hematogenous spread, medulla of lymph node is the first part to be affected. Hence periadenitis and early matting is seen in lymphatic spread. Microscopically, it presents as a central zone of caseation necrosis which stains pink with eosin surrounded by the zone of epitheloid cells with few langhan's type of giant cells. In the outermost zone, few deeply staining lymphocytes with fibrous tissue can be seen.

Baskota et al. found that level $\mathrm{V}$ lymph node was most commonly involved (51\%); while Jha et al. found upper jugular nodes (level II) to be most commonly affected, similar to our study. ${ }^{7,8}$ FNAC was found to be the most efficient technique used for diagnosis similar to findings of study by Nalini and Vinayak. ${ }^{9}$ Malakar et al. also found in their study that FNAC was sensitive to detect tuberculous lymphadenopathy in $79 \%$ patients. ${ }^{10}$

Laryngeal TB occurs mostly secondary to pulmonary 
tuberculosis or may be primarily involved too. ${ }^{11}$ In our study, it was the second most common lesion found. This agrees with the study by Choudhury et al. ${ }^{12}$

TB of middle ear cleft is very rare. Occurs primarily by hematogenous spread or secondary to pulmonary tuberculosis due to entry of bacteria through eustachian tube during cough or sneeze. ${ }^{13}$

Tuberculosis of nose is mainly secondary to pulmonary TB and presents with nasal discharge, nasal blockage, nasal ulcers or friable nasal masses which bleed easily and attached to the septum or inferior turbinate. Diagnosis of nasal TB is by histopathological examination of nasal masses. ${ }^{14} \mathrm{~TB}$ of parapharyngeal space occurring without cervical spine caries is rare. In such patients, the focus of sepsis is usually in tonsil or pharynx. The infection can affect either the prestyloid or poststyloid or both the compartments. Definitive diagnosis is by isolation of $\mathrm{M}$ tuberculosis bacterium on smear or culture. CT scan is of great help in assessing the extent of the abscess. ${ }^{15}$ Involvement of major salivary glands is uncommon and is usually secondary to pulmonary TB, but the tonsils or oral cavity may be the source of primary infection. Presentation is with local swelling, multiple fistula intraorally or extraorally, irregular low-grade fever and pain during mouth opening. Investigations for salivary gland TB include FNAC, USG, ZN staining and culture of discharge if any and salivary flow. Open biopsy is to be avoided because of danger of chronic fistula. ${ }^{16}$

TB of thyroid gland is extremely rare due to probable bactericidal action of the colloid and good vasculature of the gland. Symptoms are nonspecific and it may present with solitary thyroid nodule. Similarly, TB of thyroid gland can be diagnosed with ultrasonography, FNAC and polymerase chain reaction tests. ${ }^{17}$

\section{Conclusion}

Tuberculosis can affect almost every organ individually or in combination. The variable nature of manifestations of tuberculosis and the fact that it can mimic malignancy or other chronic granulomatous conditions make it essential to have high degree of suspicion for early diagnosis and prompt antituberculous treatment. As and when needed, appropriate investigations should be done to diagnose TB. AntiTB treatment for extra-pulmonary TB carries a good response rate.

\section{References}

1. Global tuberculosis report 2015 [Internet]. Geneva World Health Organization 2015. Available at: http://www.who.int/ tb/publications/global_report/en/. Accessed Mar 01, 2016

2. Aisenberg GM,Jacobson K,Chemaly RF, Rolston KV, Raad II, Safdar A. Extrapulmonary tuberculosis active infection misdiagnosed as cancer:mycobacterium tuberculosis disease in patients at a Comprehensive cancer centre(2001-2005). Cancer 2005;104(12):2882-87

3. Khan KA, Khan NA, Maqbool IM. Otorhinolaryngological manifestations of tuberculosis. JK Sci. 2002; 4(3):115-8

4. Two hour detection of MTB and resistance to rifampicin Cepheid International 2011. https://www.cepheid.com/en. Accessed Jul 2018

5. RNTCP national strategic plan 2017-2025. NSP draft 20172025

6. Sharma S, Sarin R, Khalid U.K, Singla N, Sharma PP, Behera D. Clinical profle and treatment outcome of tuberculous lymphadenitis in children using DOTS strategy. Indian J Tuberc. 2010; 57:4-11

7. Baskota DK, Prasad R, Sinha BK, Amatya RC. Distribution of lymph nodes in the neck in patients of tuberculous cervical lymphadenitis. Acta Otolaryngologica 2004;124(9):1095-98

8. Jha BC, Das A, Nagarkar NM, Gupta R, Singhal S. Cervical tuberculous lymphadenopathy: changing clinical pattern and concepts in management. Postgrad Med J. 2001; 77:185-7

9. Nalini B, Vinayak S. Tuberculosis in ear,nose, and throat practices :its presentation and diagnosis. Am J Otolaryngol. 2006; 27(1):39-45.doi:10.1016/j.amjoto.2005.07.005

10. Malakar D, Jajoo I, Swarup K, Gupta OP, Jain AP, Pofee VW. A clinical evaluation of fine needle aspiration cytology in the diagnosis of lymphadenopathy. Ind J Tub. 1991;38: 17-9

11. Ling L, Zhou SH, Wang SQ. Changing trends in the clinical features of laryngeal tuberculosis: a report of 19 patients. Int J Infect Dis. 2010;14(3):230-5

12. Choudhury N, Bruch G, Kothari P, Rao G, Simo R. 4 years experience of head and neck tuberculosis in a South London Hospital. JR Soc Med. 2005; 98:267-9

13. Sahn SA, Davidson PT. Mycobacterium tuberculosis infection in the middle ear. Chest 1974; 66(1):104-06

14. S Bahadur, A Thakar. Specific chronic infections. In: Michael Gleeson, editor. Scott Brown's otorhinolaryngology,head and neck surgery, 7th Ed. England: Hodder Arnold; 2008; 1458-60

15. Sriram R, Bhojwani KM. Manifestations of tuberculosis in Otorhinolaryngology practice: A retrospective study conducted in a coastal city of South India. Indian J Otolaryngol Head Neck 
Surg. 2017; 69(2):210-5

16. Dixit R, Sharma S, Nuwal P. Tuberculosis of oral cavity. Indian J Tuberc. 2008; 55(1):51-3

17. Yin TD, Wu W, Cao S, Li H. Analysis Of Misdiagnosis Of 4 Patients Of Tuberculosis Of Thyroid And Literature Review. Case Reports in Endocrinology (Internet). 2012 [cited 2015 Nov21]; 4 pages. doi:10.1155/2012/862595

18. Pandurang K, Sheno VS, Bhojwani K, Alva A, Prdead.V, et al.
Tuberculosis in head and neck in India:down but not yet dead. .J Mycobac Dis. 2014; 4(2):148.doi:10.4172/2161-1068.1000148

19. Hafeez M, Inayatullah, Ahmad I, Zakirullah. Otorhinolaryngological manifestations of tuberculosis. Pak J Med Sci. 2011; 27(4):855-7

20. Das S, Das D, Bhuyan U, Saikia N. Head and neck tuberculosis:Scenerio in a tertiary care Hospital of North Eastern India. Journal of Clinical and Diagnostic Research, 2016; 10(1):MC04-MC07. 